\title{
Notas sobre la tipología de las antologías poéticas latinas de los siglos XVI, XVII y XVIII
}

\author{
Alejandro Coroleu \\ ICREA - Universitat Autònoma de Barcelona \\ alejandro.coroleu@icrea.cat
}

Recepción: 19/07/2018, Aceptación: 28/02/2019, Publicación: 04/12/2019

\section{Resumen}

El propósito de este artículo es el de proponer un recorrido por la tradición antológica latina entre 1500 y 1740 .

Palabras clave

antologías poéticas; poesía vulgar; poesía neolatina; imitación

\section{Abstract \\ Notes on the typology of Latin poetry anthologies (16th-18th centuries) and compilations of Latin verse in the early-modern period. \\ Keywords \\ poetic anthologies; vernacular and Neo-Latin poetry; imitation}

The purpose of this article is to provide an overview on the development of anthologies 
El lector formado en las Humanidades asociará tal vez la palabra «flor» y sus derivados «florilegio» y «floresta» a los volúmenes escolares empleados en la lección de poesía. Hasta fecha reciente era frecuente, por ejemplo, utilizar en la práctica pedagógica de media Europa selecciones de textos, en su mayor parte poéticos y a menudo en latín, que acercaban a los estudiantes a los autores de la antigua Roma. De fácil manejo, desprovistos normalmente de aparato paratextual que pudiera entorpecer su consulta, estos volúmenes reunían lo más granado de las letras romanas, siguiendo frecuentemente un criterio cronológico o de acuerdo con el grado de los alumnos. A la vez que permitían enseñar las reglas básicas de gramática, prosodia y composición latinas con ejemplos — directos o, mejor, adaptados - de la tradición literaria latina, dichos tomos reflejaban y dictaban un canon literario. En una palabra, servían a un tiempo para la instrucción lingüística y para la formación del gusto literario de los jóvenes lectores a los que iban dirigidos. Con el florecimiento de las diversas tradiciones literarias nacionales en la segunda mitad del siglo XVI, la práctica de agrupar textos poéticos de diferentes autores en un solo volumen se enriquece. A las ediciones que incluyen selecciones o fragmentos de textos poéticos de la Roma clásica, se unen ahora tomos en los que figuran calas de la poesía del momento, agrupadas según criterios temáticos, genéricos o de vinculación a una escuela o tradición nacionales. ${ }^{1}$ Sin perder de vista su finalidad didáctica, las antologías quinientistas, en latín o en vulgar, responden mejor a criterios estéticos o de gusto. ${ }^{2}$ En las páginas que siguen querría proponer un recorrido por la tradición antológica latina de los siglos XVI, XVII y XVIII en Europa.

Aunque la costumbre de recopilar textos poéticos pertenecientes a diferentes autores existiera ya en la Antigüedad clásica, es a los humanistas del Renacimiento a quienes de veras debemos el desarrollo de la antología como formato y género propio. ${ }^{3}$ Dicha labor culmina en una recopilación de textos poéticos latinos menores o fragmentarios, denominada precisamente Anthologia latina, cuya edición se debe al esfuerzo del erudito holandés Pieter Burman (1668-1741), heredero de la labor filológica del humanismo. ${ }^{4}$ Concebido como un ambicioso

1. Aunque centrado en las letras inglesas, merece la pena consultar el volumen de Benedict (1996).

2. Una de estas colecciones poéticas en vulgar — publicada en Valladolid en 1605- es el tomo titulado Flores de poetas ilustres de Pedro Espinosa (1578-1650), volumen que surge al amparo de una larga y prestigiosa tradición. Tal como ha señalado Belén Molina Huete (2003: 377-78), lo más probable es que el volumen de Espinosa derivara no de un modelo único, sino de una combinación de fuentes italianas y de fuentes clásicas. Sobre las fuentes de Pedro de Espinosa véase también Espinosa (2011: 45-54).

3. En el caso de la literatura romana sabemos que el público compraba colecciones de pensamientos, de refranes, de textos de autores trágicos o de escritos de tono y contenido pornográfico. Por ejemplo, tenemos noticias — a través del testimonio de Catulo (xIv) — de una colección de poetas. 4. Se trata de la edición Poetae latini minores...curante Petro Burmanno, Leiden, 1731. Sobre la historia de la Anthologia Latina, véase Socas (2011). 
programa educativo orientado a dar al hombre un cierto tipo de cultura general a través de la interpretación e imitación de los textos de la Antigüedad grecolatina, el movimiento humanista tuvo su origen en la Italia de mediados del siglo XIV. Desde tierras italianas, y en poco más de un siglo y medio, el humanismo se extendió por toda Europa, llegando a determinar todas y cada una de las facetas de la vida intelectual del Renacimiento. Durante los siglos xv y xvI la actividad primaria del humanismo se centró en la recuperación, edición y anotación de los textos seńeros de la Antigüedad clásica. Con todo, para que el contenido real del humanismo pudiera arraigar en la cultura europea era necesaria la paulatina introducción de esos mismos estudios de humanidad en el ámbito de la enseñanza. Los últimos decenios del siglo xv fueron así testigos en Europa de la reforma, y en algunos casos de la fundación, de escuelas de latinidad y de universidades que pronto adaptaron los postulados educativos del humanismo italiano, basados en la explicación académica de los escritos más relevantes de la Antigüedad clásica. Con el cambio de centuria los autores latinos y, en menor medida, los autores griegos pasaron a formar la columna vertebral del currículum prescrito en la práctica docente. ${ }^{5}$

La elevación de poetas y prosistas de la Antigüedad clásica al rango de autoridad académica a lo largo y ancho del Continente europeo va estrechamente unida a la aparición de la imprenta. Conscientes de las posibilidades didácticas y pedagógicas de manuales de gramática y retórica, de colecciones de cartas, diálogos y discursos, así como de selecciones de poemas, impresores de varias ciudades europeas contribuyeron a la diseminación de la literatura griega y latina. La circulación impresa del legado literario de la Antigüedad y su presencia en el ambiente escolar a lo largo del siglo Xvi corren paralelas también a la difusión tipográfica de los principales escritos del humanismo italiano en latín y a su inclusión en el ámbito académico en dicho período. Junto a la explicación y anotación de escritos procedentes de la Antigüedad clásica, los autores latinos del Quattrocento italiano constituyeron asimismo objeto de lectura y comentario en aulas europeas a lo largo del siglo XVI. ${ }^{6}$

Lo enunciado hasta ahora vale sobre todo para la manera cómo la poesía latina clásica y neolatina (esto es, la poesía redactada en latín a partir de los años finales del siglo XIV) circuló en la Europa del Dieciséis. Las ediciones de poetas latinos clásicos y neolatinos italianos están ligadas principalmente a la escuela (y a la universidad) como buena parte del humanismo desde fines del siglo Xv. Por regla general, los textos publicados surgen de las prensas de hombres relacionados con la instrucción humanística. Se trata de estampadores interesados por cubrir la demanda del público local, y que trabajaban en estrecha colaboración con

5. Véase, al respecto, Rico (2002).

6. Abundante información al respecto puede encontrarse en el volumen de Goodman y McKay (1990). 
maestros y profesores faltos de ediciones individuales. El uso que se concedía a los poemas dependía, en general, del contexto en el que éstos se empleaban. Así en una escuela de provincias un modesto pedagogo podía utilizar las Silvae de Angelo Poliziano como ejercicio preliminar a la redacción de poesía por parte del estudiante, mientras que en las aulas universitarias de una gran capital un humanista de prestigio prefería valorar el texto en cuanto depósito de erudición clásica. ${ }^{7}$ Con todo, la difusión de este corpus poético no se limitó a la académica explicación de los textos, sino que incidió directamente en la literatura, latina o vernácula, de la época. Gracias a los comentarios y a las antologías nuevas generaciones de humanistas y de poetas pudieron dedicarse con mejores garantías a la lectura e interpretación de los poetas clásicos y neolatinos, pero también al cultivo de una literatura original, en romance o en latín, de acuerdo con los géneros favoritos del momento. ${ }^{8}$

De la popularidad en la Europa de la época de textos poéticos latinos dan fe las abrumadoras estadísticas, con centenares y centenares de ediciones, aparecidas a lo largo del Quinientos, de poesía latina clásica y neolatina. Su circulación impresa presenta, por lo demás, una serie de rasgos generales. Como es de esperar, ediciones de las obras de Horacio, Ovidio o del carmelita italiano Baptista Mantuanus (1447-1516) vieron la luz en centros tipográficos de primer orden como París, Basilea y Lyon, en ciudades que contaban con famosas universidades (Lovaina o Alcalá), pero también en localidades que podían alardear de poseer una importante escuela de latinidad (Sevilla y Estrasburgo). De poetas como Virgilio o Petrarca era frecuente editar el corpus poético completo, en tomos en folio profusamente comentados (piénsese, por ejemplo, en la espléndida edición de Virgilio a cargo del prefecto de estudios en el Colegio Imperial de Madrid, el jesuita español Juan Luis de la Cerda, en tres gruesos tomos publicados a partir de 1608)..$^{9}$ Otras veces se daban a la imprenta ediciones en cuarto que incluían colecciones de dos o tres poemas de un mismo autor, así como ediciones más pequeñas, en octavo, que traían textos individuales (por ejemplo, las Geórgicas virgilianas o el Bucolicum Carmen de Petrarca). Muchos de los tomos en octavo (e incluso de tamaño más reducido) eran precisamente las antologías poéticas que aquí nos ocupan, volúmenes que recogían la producción literaria de varios poetas. El formato elegido para la publicación de las antologías revela además el tipo de mercado y sobre todo el tipo de público al que iban dirigidos estos volúmenes. Se trataba por lo general de ediciones de bolsillo, más baratas, que iban destinadas no a los anaqueles de las bibliotecas eruditas y universitarias sino al público lector más común.

7. Para una tipología de las ediciones de las Silvae de Poliziano y su uso escolar, consúltese Coroleu (2014: 47-57 y 63-69).

8. Para las letras españolas véanse Alcina (1984) y Prieto (1984, I, 176-81).

9. Sobre este comentario véase Laird (2002). 
Paso ahora a centrarme en las recopilaciones poéticas en latín publicadas sobre todo a partir de la cuarta década del siglo XVI. Antes de pasar a examinar ejemplos concretos hemos de advertir que el panorama antológico latino renacentista es ciertamente muy variado. Así, en los párrafos siguientes prestaré atención primero a antologías de poetas latinos clásicos, a continuación a recopilaciones de poetas neolatinos, y finalmente a tomos que combinan ambos marcos temporales. Empecemos por las antologías de poesía latina clásica publicadas a lo largo del siglo xvi. Un criterio empleado por los antólogos de la época es el puramente cronológico. Agrupan textos poéticos (el adjetivo se entiende además en su sentido más lato) que pertenecen a un determinado período de la tradición literaria romana. Así, el editor y filólogo francés Henri Estienne publicó en Ginebra una colección de Fragmenta poetarum veterum Latinorum. ${ }^{10}$ Se trata de una antología de textos poéticos latinos arcaicos, menos difundidos o necesariamente fragmentarios. Son textos cuya extensión no había merecido una edición individual hasta la fecha o cuya importancia más relativa dentro del panorama de las letras latinas había hecho que escaparan a la atención de la primera filología humanística. No en vano, una vez editados los textos señeros de la Antigüedad clásica a finales del siglo Xv, los humanistas — sobre todo franceses y holandeses - de la siguiente centuria atendieron también a obras y autores de tradición fragmentaria o de menor calado. ${ }^{11}$

Ello puede apreciarse en otro de los patrones seguidos por los antólogos, el genérico. Así, desde principios del siglo Xvi contamos con colecciones de verso latino de género pastoril, como la edición veneciana de Nemesiano, Calpurnio Sículo y Gracio de 1534, que conoció numerosas ampliaciones y reediciones. Se trata de una antología que nace por aglomeración, es decir se trata de un volumen que recoge la producción bucólica de tres diferentes poetas que ya habían sido publicados previamente en ediciones individuales o dobles. ${ }^{12}$ Queda fuera de dichas antologías el gran bucólico latino (Virgilio) porque aquí el interés yace en la rareza, si se quiere, de los textos agrupados. Conviene recordar que estas antologías de verso bucólico en latín son las precursoras de las ediciones que los filólogos de los siglos XviII, xIX y xx agruparán bajo la denominación genérica de Anthologia latina o de Poetae latini minores (cfr. n. 4 supra).

Un tercer tipo de antología latina clásica lo constituyen aquellos volúmenes que ofrecen una reunión de autores cuyos poemas se clasifican por materias. De ellos el más famoso son las Illustrium poetarum flores de Octaviano della Mirando-

10. Fragmenta poetarum veterum Latinorum: Ennii, Acii, Lucilii, Laberii, Pacuvii, Afranii, Naevii, Caecilii, aliorum multorum, Ginebra, 1564.

11. Consúltense los capítulos al respecto en el segundo volumen de Pfeiffer (1981).

12. Poetae tres egregii: Nemesianus, Calphurnius Siculus, Gratius, Venecia, 1534. 
la. ${ }^{13}$ Publicado por vez primera por Aldo Manuzio en Venecia en 1507, este tomo conoció numerosísimas reediciones a lo largo del siglo xvI, como la de Amberes de 1544 o la de Lyon de 1559. La selección de Mirandola está dispuesta «in locos communes», cuyo índice figura al final del libro. Para Mirandola no hay duda alguna de que la lengua en la que organizar una antología poética es la latina (detalle éste que se puede apreciar por la ausencia de cualquier gentilicio en el título del volumen). El elenco de los poetas incluidos destaca por su variedad. Así poetas mayores de las letras latinas (Virgilio y Horacio) cohabitan con otros autores como Cornelio Galo, del cual muy poco material se ha conservado; poetas dramáticos comparten páginas con líricos y épicos; a autores de la Latinidad arcaica se suman poetas latinos tardíos y cristianos; incluso escritores a los que nos costaría denominar poetas como Petronio y Boecio merecen la atención del antólogo. El término «poeta» se toma, pues, en su sentido más amplio.

En la antología de Mirandola cada una de las entradas presenta una estructura muy semejante. Fijémonos, por ejemplo, en la voz «ambitio». A una frase sentenciosa (pág. 49: «ambitio avaritiae nutrix est, quae potentum vestibulis excubat») siguen versos de Claudiano, Plauto, Séneca y Virgilio, precedidos cada uno de ellos de una explicación. Así, al verso 37 del primer libro de las Geórgicas virgilianas («nec tibi regnandi veniat tam dira cupido») le precede la aclaración «ambitiosorum adminitio lepidissima, ut a regnandi cupiditate abstineant». La antología de Mirandola posee, por tanto, una finalidad didáctica o moralizadora, que no la hace demasiado diferente de otras colecciones — por ejemplo, de refranes— del momento.

Un segundo grupo de antologías lo constituyen las selecciones de poesía neolatina. ${ }^{14}$ También existen en estos casos diversos tipos de antologías. Las hay, por ejemplo, que agrupan poemas redactados para una ocasión especial o que celebran un acontecimiento señalado, como el nacimiento de un príncipe heredero, la muerte de un papa o una victoria militar (como en el caso de los Poemata varia, Venecia, 1572, de los hermanos Guerra para celebrar la batalla de Lepanto). Que sepamos el primer ejemplo de este subgénero es un volumen publicado en 1524 con el título de Corycana. ${ }^{15}$ Agrupa versos latinos de unos 120 poetas, todos ellos vinculados a la corte pontificia de León X, dedicados exclusivamente a la Virgen en conmemoración de una estatua de la Madre de Dios que el editor Johann Goritz había mandado erigir en su jardín romano. En este tipo de antologías es habitual la inclusión de poemas en lengua vulgar. Así, en el tomo

13. Para el presente trabajo hemos maneado la siguiente edición: Octaviano della Mirandola, Illustrium poetarum flores, Lyon, 1559.

14. Aunque descuida el panorama hispánico, el mejor análisis al respecto es el que ofrece Sparrow (1976).

15. Johann Goritz, ed., Corycana, Roma, 1524. 
Tempio a Giovanna d'Aragona (Venecia, 1554), editado por Girolamo Ruscelli para conmemorar la construcción en Roma de un templo en honor a Juana de Aragón, figuran 118 páginas de poemas latinos, junto a poesías en italiano (388 páginas), castellano (15 páginas) y griego antiguo (10 páginas).

En algunos casos la temática religiosa o amatoria guía la selección y recopilación de ciertas composiciones. Fijémonos, por ejemplo, en una curiosa edición de textos poéticos italianos en latín que se publica en Alcalá de Henares en $1534 .{ }^{16}$ Además de versos de carácter más laico, en un mismo volumen se edita el De partu Virginis del italiano Jacopo Sannazaro con la versificación del Evangelio de Arator como polos opuestos de un ideal del humanismo cristiano renacentista. El volumen incluye también textos de carácter más laico como las Eclogae del propio Sannazaro, una pieza de la poetisa Cassandra Marchiesi y el poemita de Pietro Bembo Benacus. Cierra el libro un epigrama recomendando la lectura de Arator del profesor complutense Ramiro de Daroca. La sorprendente mezcla de poemas eróticos y composiciones religiosas puede justificarse por la voluntad de ofrecer variedad formal y por la probada religiosidad de los autores incluidos. Como contrapunto debemos mencionar, siquiera brevemente, tres colecciones de verso amatorio, agrupadas bajo el nombre de Horti tres amoris amoenissimi y publicadas en 1567 por Aegidius Periander. ${ }^{17}$ Se trata de una antología mixta, que aúna criterios genéricos y de nacionalidad: versos eróticos de poetas neolatinos italianos, franceses y alemanes, a los que el antólogo asigna el nombre de un determinado árbol, pájaro o flor.

Contamos también con colecciones que presentan toda la producción poética de un puñado de autores. En muchos casos el impresor escoge una media docena de poetas contemporáneos cuyo corpus poético no es demasiado abundante y reproduce casi la totalidad de lo que aquéllos han escrito. El prototipo son los Carmina quinque illustrium poetarum (Bembo, Navagero, Castiglione, Cotta y Flaminio), publicados en Venecia en 1548 por Vincenzo Valgrisi. ${ }^{18}$ Cabe destacar que Valgrisi actúa como un verdadero editor toda vez que muchos de los poemas aparecen reproducidos aquí por vez primera, habiendo circulado anteriormente sólo en versión manuscrita. El antólogo hace, pues, las veces de filólogo, fija en definitiva el texto.

A lo largo del siglo xvı encontramos asimismo antologías que contienen selecciones de las obras de los autores representados. Los adjetivos que figuran en las portadas de dichas ediciones (illustres, nobiles, praestantes, calificativos que denominan a los poetas, no a los poemas) indican ya cuáles son los criterios y principios por los que se guían los compiladores. Su finalidad era la de ofrecer una selección

16. Pia et emuncta opuscula. Iacobi Synceri Sannazarii..., Alcalá de Henares, 1534.

17. El título completo es Horti tres amoris amoenissimi, praestantissimorum poetarum nostri saeculi, flosculis et plantulis odoferis iam primum ab Aegidio Periandro consiti, Frankfurt, 1567.

18. Vincenzo Valgrisi, ed., Carmina quinque illustrium poetarum, Venecia, 1548. 
lo más representativa posible de la poesía latina de personas que habían alcanzado fama en cuanto poetas, filólogos o eruditos. Aunque a veces dichas compilaciones incluían también poemas anónimos o de autoría incierta, sus contenidos los determinaba casi exclusivamente la elección de ejemplos de la producción poética de un autor importante. En estos volúmenes los poemas se agrupaban no según su género o tema, sino por autor. Rara vez se presentaban los poetas cronológicamente. El orden es, las más de las veces, arbitrario y a la contribución de cada autor no acompañan nunca datos biográficos u observaciones críticas. El volumen se presenta más como una antología de poetas que como una antología de poemas. La más extensa colección de este tipo fue publicada en París por Léger Duchesne en dos tomos en décimo sexto, en 1555 y 1560, titulados respectivamente Flores Epigrammatum y Farrago Poematum, este último en alusión al forraje — farrago - para los animales constituido por la mezcla de diversos granos. Ambos tomos recogen muestras de poesía neolatina, sobre todo italiana, «ex optimis quibusque authoribus excerpti». ${ }^{19}$

Un último apartado lo constituyen las antologías nacionales, que agruparían parte de la producción poética de un determinado país. Conviene aclarar que ninguna de estas colecciones aspira a ser exhaustiva o a representar los logros poéticos de toda una nación. De todas las antologías que merecen tal nombre la primera es los Carmina illustrium poetarum Italorum, publicada en dos volúmenes en París en 1576 y 1577 por Giovanni Matteo Toscano. ${ }^{20}$ En el prólogo a su edición, dirigido a los «esforzados lectores de poesía», Toscano explica las razones que le han llevado a editar su libro. La poesía, señala, pasa por un mal momento por culpa de la imprenta (fol. $1 \mathrm{v}$ : «ars typographica... huic calamitati ansam praebuit»). Toscano acusa a los editores de falta de gusto y de criterio estético y de sólo buscar el beneficio económico. A menudo la mejor poesía permanece inédita. Por ello, Toscano ha decidido seleccionar ejemplos ilustres de entre toda la poesía latina que ha leído o que sus amigos le han proporcionado y ha preparado el volumen que el lector tiene en sus manos. Deliberadamente ha optado por ceñirse al área italiana dejando para futuros antólogos la selección de verso latino francés y, significativamente, español. El orden empleado por Toscano para su selección es misceláneo y caótico, pero ello no fue óbice para el libro gozara de un éxito sin precedentes, suscitando a la vez admiración y plagio. ${ }^{21}$

Examinemos, por último, los contenidos de tres antologías que reúnen muestras de poesía latina clásica y de poesía neolatina. La primera de ellas aparece publicada en la Florencia de los Medici, en 1504, al cuidado del editor Benedetto Filologo. Se trata de una antología organizada según un criterio genérico y dia-

19. Léger Duchesne, ed., Flores Epigrammatum (París, 1555) y Farrago Poematum (París, 1560).

20. Giovanni Matteo Toscano, ed., Carmina illustrium poetarum Italorum, París, 1576 y 1577.

21. Véase Sparrow (1976: 396-97). 
crónico. El volumen incluye los versos de Virgilio, calas de poesía latina pastoril menor y ejemplos de églogas renacentistas, como el Bucolicum Carmen de Petrarca, las bucólicas de Boccaccio o los versos de Battista Mantuano, el Virgilio cristiano, además de textos del napolitano Pomponio Gaurico (1482-1530). ${ }^{22}$ Del carácter escolar del tomo dan cuenta los comentarios del propio Benedetto —en el prólogo a su compilación- a propósito de los contenidos del poema petrarquesco y de la poesía latina de Boccaccio. El editor advierte a su joven destinatario que las églogas de ambos autores tratan temas «que los oídos doctos y limpios rechazarán completamente». Con todo, ambos poemas son dignos de imitación literaria y, a pesar de sus propias reservas, Benedetto recomienda los textos «ad latinae elegantiae studiosum». ${ }^{23}$

El volumen de Benedetto delinea, si se quiere, el progreso de la poesía bucólica latina desde los días de Virgilio hasta los autores contemporáneos del propio editor. A su vez servirá como fuente de inspiración para antologías posteriores, algunas ya mencionadas que se centran en la bucólica antigua, y otras que abarcan incluso buena parte de la poesía pastoril literaria renacentista. De entre estas últimas cabe destacar una edición publicada por Johannes Oporinus en 1546 que recoge varios siglos de poesía bucólica en latín. ${ }^{24}$ Se trata de una colección sumamente original ya que en el prólogo el editor reflexiona sobre la propia historia literaria del género bucólico. Es singular también en la medida en que la antología combina el criterio cronológico y la ordenación temática. Así, los autores de la Antigüedad, salvo Virgilio, vienen primero, seguidos por poetas bucólicos de inspiración cristiana y por ejemplos de poesía pastoril de tono más moralizador. Al final se incluyen calas de poesía bucólica de corte amoroso, siempre más moderna.

Al igual que la compilación de Oporinus, la última antología a la que nos referiremos no establece tampoco distinción alguna entre la poesía latina redactada en la Antigüedad y la producción poética del humanismo latino. Se trata de una selección publicada por el jesuita tarraconense Josep Morell en 1684. Para Morell —que enseñó letras humanas en Tarragona, donde bien pudo haber prescrito su volumen como manual de ejercicios de composición latina- la imitación literaria es además un proceso que transciende cualquier límite temporal:

[al ingenioso lector] Hallándome por obediencia ocupado en la enseñanza de las letras humanas determiné con todas veras aplicarme al ejercicio de ellas... No sigo

22. Sobre la fama de Battista Mantuano en la Europa del siglo Xvi, véase Piepho (2001).

23. Eclogae. Vergilii. Calphurnii. Nemesiani. Francisci Petrarchae. Ioannis Boccaccii. Ioan. Bap. Mantuani. Pomponii Gaurici, Florencia, Filippo di Giunta, 1504, f. 1r: «Iam ad opus accedamus, in quo illud praefari ducimus, quod in eclogis Francisci Petrarchae et Ioannis Boccaccii nonnulla deprehenduntur quae doctae et purgatae aures penitus respuent».

24. En habes lector Bucolicorum autores XXXVIII quotquot videlicet a Vergilii aetate ad nostra usque tempora, Basilea, Oporinus, 1546. 
orden de tiempo en los autores cuyas poesías traduje, antes bien pongo en primer lugar los católicos, dejando para el último los gentiles, aunque más antiguos. ${ }^{25}$

El jesuita incluye así en su compilación, junto a poemas de Marcial y Horacio, a varios poetas neolatinos, como el napolitano Giovanni Pontano, su compañero de congregación, el francés Remond, y un poeta en latín, Jaime Falcó, elogiado por Baltasar Gracián en su Agudeza y arte de ingenio (xIx. 324) como «el Marcial de Valencia». La presencia, en la colección de Morell, de un poeta como Marcial, autor gnómico por excelencia, y de poetas religiosos y morales apunta a una antología concebida con fines edificantes, toda vez que los textos recogidos aparecen en traducción.

El volumen del jesuita tarraconense incluye, además, por igual calas de poetas romanos y neolatinos, pero siempre en traducción, aspecto éste que no debería sorprendernos. Numerosos poetas de los siglos XVI y XVII utilizaron sólo la lengua vernácula. Pero no faltan tampoco autores que emplearon vulgar y latín de modo indistinto, o que escogieron únicamente la lengua latina como vehículo de expresión poética. Con todo, incluso aquellos poetas que se inclinaron exclusivamente por el castellano se habían formado en la imitación de los poetas clásicos latinos y de la poesía neolatina en las aulas escolares. En la escuela se habían ejercitado en la redacción de poemas en latín y en la traducción de versos latinos a la lengua vulgar, o habían aprendido a traducir del romance al latín. De ahí, por ejemplo, la costumbre de incluir en selecciones de poemas, cual si se tratara de una poesía original más, traducciones de poetas clásicos latinos. El propio Pedro Espinosa lo hace en sus Flores con los versos de Horacio. No en vano, el antólogo antequerano recalca la importancia de las versiones horacianas en el prólogo, al tiempo que la traducción de las odas se anuncia en la cubierta del volumen. ${ }^{26}$

Conviene recordar que los modelos renacentistas en latín (a la vez que los géneros latinos clásicos) constituyeron la raíz de la abundante poesía neolatina del Renacimiento y Barroco europeos, y contribuyeron a la creación de una expresión poética en la lengua vulgar. Paradójicamente, la consolidación, después de 1550, de la poesía vernácula coincidió con la gran eclosión de poesía neolatina, y a partir de entonces la poesía del humanismo europeo, fuera cual fuera su lengua de expresión, presentó siempre amplias zonas de confluencia, acentuándose esta conexión hacia finales del siglo XVI. Fueran autores de poesía original o traductores, escribieran en latín o en vulgar, los poetas de los siglos XVI y XVII accedieron a los modelos clásicos y neolatinos a través de la práctica en el ámbito escolar y universitario, por medio de ediciones anotadas o, en numerosos casos, de las antologías que aquí hemos examinado.

25. Poesías selectas de varios autores latinos, Tarragona, 1684, 7. Sobre la antología de Morell, véase Taylor (2006, 161-62).

26. Pedro Espinosa, Flores de poetas ilustres de España, Valladolid, 1605, 6v: «De paso advertid que las odas de Horacio son tan felices que se aventajan a sí mismas en su lengua Latina». 


\section{Apéndice}

Ofrecemos en este apéndice un listado, en absoluto exhaustivo, pero sí lo suficientemente significativo, de las antologías de poesía latina publicadas en Europa entre 1500 y 1740 . Nos ha parecido que merecía la pena incluirlas aquí como muestra de la pervivencia del género hasta bien entrado el siglo xviII. Cuando lo hay, indicamos la signatura de un ejemplar localizado en una biblioteca española.

\section{Antologías latinas clásicas}

Poetae tres egregii: Nemesianus, Calphurnius Siculus, Gratius, Venecia, Aldo Manuzio, 1534.

Octaviano della Mirandola, Illustrium poetarum flores, Lyon, Apud Ioannem Tornaesium \& Gul. Gazeium, 1559 (Santander, Biblioteca de Menéndez Pelayo, 517).

Fragmenta poetarum veterum Latinorum: Ennii, Acii, Lucilii, Laberii, Pacuvii, Afranii, Naevii, Caecilii, aliorum multorum, Ginebra, Henri Estienne, 1564 (BNE, R/22099).

\section{Antologias neolatinas}

Johann Goritz, ed., Coryciana, Roma, Ludovicus Vicentinus, 1524 (BNE, $\mathrm{R} / 20245-2)$.

Pia et emuncta opuscula. Lacobi Synceri Sannazarii..., Alcalá de Henares, Miguel de Eguía, 1534 (Santander, Biblioteca de Menéndez Pelayo, 12.555).

Vincenzo Valgrisi, ed., Carmina quinque illustrium poetarum (Bembo, Navagero, Castiglione, Cotta y Flaminio), Florencia, Torrentinus, 1548 (BNE, R/19693).

Girolamo Ruscelli, ed., Tempio a Giovanna d'Aragona, Venecia, per Plinio Pietrasanta, 1554 (BNE, R/17187).

Léger Duchesne, ed., Flores Epigrammatum y Farrago Poematum, París, De Marnef, 1555 y 1560 (BNE, u/7638-9).

Horti tres amoris amoenissimi, praestantissimorum poetarum nostri saeculi, flosculis et plantulis odoferis iam primum ab Aegidio Periandro consiti, Frankfurt, Huter, 1567.

In Foedus et victoriam contra Turcas... poemata varia, Venecia, Typogr. Guerraea, 1572.

Giovanni Matteo Toscano, ed., Carmina illustrium poetarum Italorum, París, Gorbinus, 1576 y 1577 (BNe, 5/4080).

Jan Gruter, Delitiae poetarum Italorum, Frankfurt, Rosa, 1608 (Madrid, Biblioteca del Palacio Real, Ix/4596).

Francis Atterbury, Anthologia, seu selecta quaedam poemata Italorum qui latine scripserunt, Londres, R. Green \& F. Hicks, 1684 (BNE, 2/20458).

Selecta poemata Italorum qui latine scripserunt, iterum in lucem data, una cum aliorum Italorum operibus, accurante A. Pope, Londres, J. \& P. Knapton, 1740. 
Antologías latinas de verso clásico y neolatino

Eclogae. Vergilii. Calphurnii. Nemesiani. Francisci Petrarchae. Ioannis Boccaccii. Ioan. Bap. Mantuani. Pomponii Gaurici, Florencia, Filippo di Giunta, 1504 (BNE, 2/21598).

En habes lector Bucolicorum autores XXXVIII quotquot videlicet a Vergilii aetate ad nostra usque tempora, Basilea, Oporinus, 1546 (BNE, 2/69505).

Poesías selectas de varios autores latinos. Traducidas en verso castellano e lustradas con notas de la erudición que encierran, por el padre Joseph Morell religioso de la Compañia de Jesús, Tarragona, Joseph Soler, 1683 y 1684 (Biblioteca Universitària de Barcelona, C-186/5/24). 


\section{Bibliografía}

Alcina, Juan Francisco, «Entre latín y romance: modelos neolatinos en la creación poética castellana», eds. J. Ma Maestre Maestre y J. Pascual Barea, Humanismo y pervivencia del mundo clásico, Cádiz, Universidad de Cádiz, 1993, I, 3-27.

Benedict, Barbara M., Making the Modern Reader: Cultural Mediation in early modern Literary Anthologies, Princeton, Princeton University Press, 1996.

Coroleu, Alejandro, Printing and Reading Italian Latin Humanism in Renaissance Europe (ca. 1480 - ca. 1540), Newcastle upon Tyne, Cambridge Scholars Publishing, 2014.

Espinosa, Pedro, Flores de poetas ilustres de España, Luis Sánchez, Valladolid, 1605.

—, Poesía, edición, introducción y notas de Pedro Ruiz Pérez, Madrid, Castalia, 2011.

Goodman, Anthony y Mccay, Angus, eds., The Impact of Humanism on Western Europe, Londres, Longman, 1990.

LaIrd, Andrew, "Juan Luis de La Cerda, Virgil, and the Predicament of Commentary», The Classical Commentary: History, Practices, Theory, eds. Roy K. Gibson y Christina Shuttleworth Kraus, Leiden, Brill, 2002, 171-203.

Molina Hiete, Belén, La trama del ramillete. Construcción y sentido de las "Flores de poetas ilustres» de Pedro Espinosa, Sevilla, Fundación José Manuel Lara, 2003.

Pfeiffer, Rudolf, Historia de la filología clásica, Madrid, Gredos, 1981.

Piepho, Lee, Holofernes' Mantuan: Italian Humanism in Early Modern England, Nueva York-Oxford, Peter Lang, 2001.

Prieto, Antonio, La poesía española del siglo XVI, Madrid, Cátedra, 1984.

Rico, Francisco, El sueño del humanismo: de Petrarca a Erasmo, Barcelona, Destino, 2002.

Socas, Francisco, «Desguace y restauración de la Anthologia Latina», Paulo minora: Estudios de poesia latina menor y fragmentaria, eds. José Luis Vidal, José Ignacio García Armendáriz y Adolfo Egea, Barcelona, Publicacions i edicions de la Universitat de Barcelona, 2011, 17-48.

Sparrow, John, «Renaissance Latin Poetry: Some sixteenth-century Italian Anthologies», Cultural Aspects of the Italian Renaissance. Essays in Honour of Paul-Oskar Kristeller, ed. Cecil. H. Clough, Manchester, Manchester University Press, 1976, 386-405.

TAYLOR, Barry, «Iberian-Latin Bilingual Editions, Fifteenth-Eighteenth Centuries», Latin and Vernacular in Renaissance Iberia, II: Translations and Adaptations, eds. Barry Taylor y Alejandro Coroleu, Manchester, Manchester Spanish and Portuguese Studies, 2006, 149-69. 
